\title{
The Role of the Microbiota in Inflammatory Disease: The Celiac Disease Model.
}

\author{
Rituparna De* \\ Postdoctoral position at Department of Radiation Sciences, Umea University, Umea, Sweden
}

Accepted on May 17, 2018

\section{Introduction}

Celiac disease (CD) is a life-threatening enteropathy which follows an autoimmune etiology. The CD victim is found to develop sensitivity to gluten and related prolamines. CD pathogenesis is multifactorial, and genetic predisposition due to possession of HLA-DQ2 and or HLA-DQ8 alleles of the Major Histocompatibility Complex (MHC) loci is a primary cause. It is a severely debilitating inflammatory disease and the primary treatment includes withdrawal of gluten from the diet. The clinical pathology includes intraepithelial lymphocytosis and influx of lymphocytes to the lamina propria leading to villous atrophy and crypt hyperplasia due to an overproduction of the pro-inflammatory cytokine IFN- $\gamma$. Recent studies by advanced techniques have found an important association between CD pathogenesis and dysregulation of multiple immune regulatory pathways [1].

Celiac disease is widespread in the western hemisphere and gradually emerging as a threat in the east with a prevalence of $1 \%$ in both the regions of the world. $90 \% \mathrm{CD}$ patients are reported to carry the HLA-DQ2/HLA-DQ8 alleles. $40 \%$ of the western population possess this genotype however only $1 \%$ develop the disease. This led to the speculation and discovery of other factors responsible for causing the disease. These include environmental, epigenetic and non-HLA genetic factors and association of dysbiosis of the gut microbiota. It is believed that a complex interaction among these components leads to disease manifestation.

Dysbiosis of microbiota is a frequently observed phenomenon in $\mathrm{CD}$ patients and recent reports indicate a crucial role of the microbiota in $\mathrm{CD}$ pathogenesis. A study reported by Caminero et al. found that certain gut-associated bacteria like $P$. aeruginosa and Lactobacillus $s p$. has the capacity to modify gluten peptides and enhance or reduce their immunogenicity and potential to activate T-cells, thereby modulating risk of CD in genetically susceptible individuals [2]. Tian et al. who studied the oral microbiota in patients with active and refractory $C D$ and compared them with that of healthy controls reported the increased number of salivary Lactobacillus sp. and suggested this was responsible for higher gluten-degrading activity of CD subjects as opposed to normal subjects [3]. Gutierrez et al., studied from fecal samples of CD patients versus normal subjects the quantification and contribution of glutenase involved in degrading gluten and concluded from their study that microbial activity can modify digestion of the remaining fraction of 33-mer peptides that result from gluten digestion in humans [4]. These findings suggest the significant role played by oral and gut-associated bacteria in enzymatically degrading gluten to immunogenic forms which provide the immunogenic trigger in CD subjects.

Recent studies have found a link between infection exposure and CD pathogenesis. For example, Candida albicans infection has been proposed as a factor involved in stimulating the onset of CD in genetically predisposed individuals [5]. There has been constant debate whether or not Helicobacter pylori infection affects CD onset and pathogenesis [6]. More studies have reiterated the assumption that the microbiota is involved as cause or consequence of $\mathrm{CD}$ and clearly demonstrated the role of the microbiota and dysbiosis of the microbiome in association with $\mathrm{CD}$ and which contribute mainly in steps of gluten digestion and degradation, modulating the outcome of the clinical impact of gluten either by augmenting or abrogating the symptoms. More research and a deeper understanding of the complex connection between microbiota and innate and adaptive immunity network can resolve the problems of CD prevention and outcome and pave new paths for novel therapeutic approach. Enrichment or depletion of required microbial components that contribute to pathogenesis in $\mathrm{CD}$ can be a useful tool to combat the pathophysiology of CD. For example, $P$. aeruginosa isolated from human $\mathrm{CD}$ cases when injected into mice duodenum exhibited elastase activity that degraded gluten into peptides which were depicted to trigger gluten-specific $\mathrm{T}$ cells [2]. Reducing the load of this bacteria may induce tolerance in CD subjects. On the other hand, Lactobacillus sp. from non-CD subjects degraded peptides that resulted from gluten digestion due to human and $P$. aeruginosa proteases. These approaches can be used to deal with CD therapy in a patient-specific manner as these can alleviate the need for many CD subjects to be prescribed a totally GFD restriction. Probiotic use can be an additional treatment for $\mathrm{CD}$ patients and researchers have demonstrated the efficacy of probiotic bacteria in alleviating the symptoms of gluten intolerance. Gallpeau et al. showed that elafin (a serine protease inhibitor produced by small intestinal epithelial cells) expression in the small intestinal epithelium was lower in patients with active CD compared with control patients without $\mathrm{CD}$. In in vitro experiments, elafin significantly slowed the kinetics of the deamidation of the 33-mer peptide to its more immunogenic form. Treatment of gluten-sensitive mice with elafin was delivered to the intestine of the glutensensitive mice by the Lactococcus lactis vector normalized inflammation, improved permeability, and maintained ZO-1 expression [7]. McCarville et al. showed a commensal strain of Bifidobacterium longum that produces serine protease inhibitor can be used to attenuate gliadin-induced immunopathology and influences microbiota composition in NOD/DQ8 mice [8]. 


\section{References}

1. Dieli-Crimi R, Cenit MC, Nunez C. The Genetics of Celiac Disease: A comprehensive Review of Clinical implications. J Autoimmun. 2015;64:26-41.

2. Caminero A, Galipeau HJ, McCarville JL, et al. Gastroenterology. 2016; 151(4):670-683.

3. Tian N, Faller L, Leffler DA, et al. Salivary Gluten Degradation and Oral Microbial Profiles in Healthy Individuals and Celiac Disease Patients. Appl Environ Microbiol. 2017;83(6):e03330-16.

4. Gutierrez S, Andres JP, Blanco HM, et al. The Human Digestive Tract has Proteases Capable of Gluten Hydrolysis. 2017;6(7):693-702.

5. Corouge M, Loridant S, Fradin C, et al. Humoral Immunity
Links Candida albicans Infection and Celiac Disease. 2015;10(3)

6. Dore MP, Salis R, Loria MF, et al. Helicobacter Pylori Infection and Occurrence of Celiac Disease in Subjects Hla-Dq2/Dq8 Positive: A prospective study Helicobacter. 2018;23(2):e12465.

7. Galipeau HJ, Wiepjes M, Motta JP, et al. Novel Role of the Serine Protease Inhibitor Elafin in Gluten-Related Disorders. Am J Gastroenterol. 2014;109(5):748-756.

8. McCarville JL, Dong J, Caminero A, et al. A Commensal Bifidobacterium Longum Strain Improves Gluten-Related Immunopathology in Mice Through Expression of a Serine Protease Inhibitor. Appl Environ Microbiol. 2017:0132301417.

\section{*Correspondence to:}

Rituparna De

Department of Radiation Sciences,

Umea University,

Umea, Sweden

Tel: +46(0)700059384

E-mail: rituparna26@gmail.com 